\title{
Study on Comparative Diagnostic Efficacy of HSG \& Laparoscopy in Infertility
}

\author{
Shyamali Duttaํㅗ Palash Mazumder², Dimple Mishra³ , Jhantu Kumar Saha ${ }^{4}$
}

\begin{abstract}
${ }^{1}$ Department of Obstetrics and Gynaecology, Medical College, Kolkata, West Bengal, India. ${ }^{2}$ Department of Obstetrics and Gynaecology, Medical College, Kolkata, West Bengal, India. ${ }^{3}$ Department of Obstetrics and Gynaecology, Medical College, Kolkata, West Bengal, India. ${ }^{4}$ Department of Obstetrics and Gynaecology, Medical College, Kolkata, West Bengal, India.
\end{abstract}

\section{ABSTRACT}

\section{BACKGROUND}

Tubal occlusion is one of the most frequent causes of infertility in women. The evaluation of the fallopian tube is necessary in female infertility. The two most important diagnostic procedures for evaluation of tubal patency are hysterosalpingography (HSG) and laparoscopy. We wanted to compare the diagnostic efficacy of HSG \& diagnostic laparoscopy in evaluation of tubal patency in infertility.

\section{METHODS}

A hospital OPD based prospective study was conducted among fifty patients fulfilling the inclusion and exclusion criteria who attended the OPD for treatment of infertility in the Department of Obstetrics and Gynaecology, Medical College and Hospital, Kolkata from $1^{\text {st }}$ January 2016 - 31 ${ }^{\text {st }}$ December 2017. After taking proper history, thorough examination and basic investigations for infertility work-up, HSG and Laparoscopy were performed.

\section{RESULTS}

Analysis showed that most of the patients were in the age group of 30 to 35 years. Incidence of primary and secondary infertility was $64 \%$ and $36 \%$ respectively. Right proximal and distal blockage in HSG, and DL were 56\% and $40 \%$ in HSG and $48 \%$ and $28 \%$ in DL respectively. $\mathrm{p}=0.0001$. Left proximal and distal blockage in HSG and DL were $32 \%$ and $56 \%$ in HSG and $40 \%$ and $28 \%$ in DL. Right and left hydrosalpinx on HSG and DL were $32 \%$ and $12 \%$ in HSG and $40 \%$ and $16 \%$ in DL. p $<0.005$. Right and left peri-tubal adhesions on HSG and DL were $36 \%$ and $20 \%$ at HSG and $52 \%$ and $28 \%$ in DL. $\mathrm{p}<0.005$. Thus, laparoscopy is superior in detection of hydrosalpinx and peritubal adhesions than HSG. In this analysis, the incidence of submucosal, intramural and subserous fibroids was $16 \%, 12 \%$ and $8 \%$ respectively. Incidence of septate, unicornuate, bicornuate \& arcuate uterus was $8 \%, 6 \%, 6 \%, 2 \%$ respectively. Intrauterine adhesions were detected in $12 \%$ and endometrial polyp in $4 \%$ of patients.

\section{CONCLUSIONS}

HSG is considered to have a high sensitivity and specificity to detect tubal block. HSG and laparoscopy are not alternative, but are complementary in the evaluation of tubal block.

\section{KEY WORDS}

Infertility, Tubal Occlusion, Diagnostic Accuracy, Hysterosalpingography, Laparoscopy
Corresponding Author: Dr. Palash Mazumder, Akshara Lotus Garden, Block No. 3, Flat 1A. F/F2, Hatiara Road, Kolkata-700159, West Bengal, India.

E-mail:drpalash2011@gmail.com

DOI: $10.14260 / \mathrm{jemds} / 2020 / 202$

Financial or Other Competing Interests: None.

How to Cite This Article:

Dutta S, Mazumder P, Mishra D, et al. Study on comparative diagnostic efficacy of hsg \& laparoscopy in infertility. J. Evolution Med. Dent. Sci. 2020;9(12):937942, DOI: $10.14260 /$ jemds/2020/202

Submission 12-01-2020,

Peer Review 26-02-2020,

Acceptance 04-03-2020,

Published 23-03-2020.

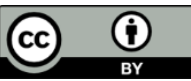




\section{BACKGROUND}

Infertility is an emerging \& psychologically devastating problem for the couples. ${ }^{1}$ Infertility \& childlessness is one of the important \& underappreciated reproductive health problems in developing countries. The inability to procreate is frequently considered as a personal tragedy \& a curse for the couples, implicating on entire family \& even on the local community. Negative psychological consequences of childlessness are common \& often severe. Infertility is "a disease of the reproductive system defined by the failure to achieve a clinical pregnancy after 12 months or more of regular unprotected sexual intercourse." (WHO-ICMART glossary 2009). Infertility affects up to $12-28 \%$ of reproductive aged couples worldwide. ${ }^{2}$ Male $\&$ female factors accounts for $20-30 \%$ \& 20-35 \% cases of infertility respectively. ${ }^{3}$ Tubal factors cause $25-30 \%$ of infertility cases. ${ }^{4}$ Tubal blockage is one of the main causes of infertility. In the routine work-up, our ability to evaluate tubal function is limited to tubal patency \& peritubal adhesions. The primary cause of tubal infertility is its blockage ${ }^{5,6}$ mainly due to pelvic inflammatory diseases. Other causes include severe endometriosis, adhesions from previous tubal \& nontubal surgery \& pelvic tuberculosis. Program for investigating infertile patients include a variety of tests: physical examination, laboratory testing \& most of the time, radiological \& surgical studies.

Determining the presence \& absence of tubal occlusion has both prognostic \& therapeutic importance. At present time HSG, sonohysterosalpingography, hysteroscopy \&laparoscopy are widely accepted screening procedures enabling the effective assessment of both tubal patency \& uterine cavity. In such patients the diagnostic workup should start with HSG which is a very useful initial screening test. Hysterosalpingography (HSG) \& laparoscopy are the two classic methods for evaluation of tubal patency in infertile women \& are complementary to each other, having both advantages \& disadvantages. Both methods are accessible \& affordable in many hospitals. The HSG is a contrast enhanced fluoroscopic and flat plate study performed by instilling radio opaque dye into the uterine cavity through a cannula that evaluates the uterine cavity \& reveals the internal architecture of the tubal lumen. HSG is best scheduled during the 6-10 days of menstrual cycle. The endometrium is thin during this proliferative phase, a fact that facilitates image interpretation and should also ensure that there is no pregnancy. This is minimally invasive \& considered as the first line approach of evaluating tubal patency.

Laparoscopy is regarded generally as the definitive test for the evaluation of tubal factors. It is inspection of the pelvic cavity through a cold light endoscope pass through the abdominal wall under anaesthesia. During this procedure a dye is injected through a cannula in uterine cervix to test the patency of the fallopian tubes. This investigation is now frequently performed, but it does carry potentially serious risks like perforation of viscous, haemorrhage due to blood vessel damage or a trocar puncture. This technique only indicates patency of entire utero-tubal cavity \& does not provide information concerning the location of the potential abnormality. Although it is a time taking \& invasive procedure, done under GA, laparoscopy has major advantages. It provides both a panoramic \& magnified view of the pelvic reproductive anatomy. Consequently, it can identify milder degree of distal tubular occlusive diseases, pelvic \& adnexal adhesions \& endometriosis that adversely affect fertility but escape by HSG. Most importantly, Laparoscopy offers the opportunity to treat disease at the time of diagnosis like lysis of filmy or focal adhesions \& excision or ablation of superficial endometriosis. Laparoscopy is better predictor of future fertility than HSG.

Laparoscopy is considered as the clinical reference test for diagnosing tubal pathology ${ }^{7} \&$ it provides information about the pelvic anatomy including peri-adnexal adhesions, endometriosis \& ovarian pathology which cannot be done with HSG. ${ }^{8}$ False negative \& false positive results though uncommon but do occur, particularly where fallopian tubes are obscured by adhesions. Whereas tubal obstructions detected by HSG are frequently not confirmed at laparoscopy, patency almost always is.

We wanted to compare the diagnostic efficacy of HSG \& diagnostic laparoscopy in evaluation of tubal patency in infertility.

\section{METHODS}

This was a Hospital OPD based Prospective Study. The study protocol was approved by the institutional ethics committee, Medical College, Kolkata \& the study was performed at the department of Obstetrics \& Gynaecology. Patients were enrolled after taking their informed written consent. Participants were assured that their information would be kept confidential. All patients who have been clinically diagnosed of infertility aged between 20-35 years \& posted for diagnostic laparoscopy 3 months after doing HSG were included in the study. Patients with prior pelvic surgery, ovulatory dysfunction, abnormal bimanual pelvic examination, active pelvic inflammatory disease \& cervical pathologies like polyp \& stenosis, hypersensitivity to contrast, associated medical illness like hypertension, diabetes mellitus \& epilepsy, gross abnormality in husband semen analysis were excluded. Study Period was $1^{\text {st }}$ January 2016 - 31 ${ }^{\text {st }}$ December 2016.

Sample Size was fifty (50) patients (100 results as HSG and Laparoscopy were done for the same patients) randomly selected from OPD (G\&O). This sample size was determined based on the cost, time \& convenience of data collection. It is proportionate of population concerned having sufficient statistical power, as determined by different previous studies. All eligible patients were examined thoroughly \& history was taken properly. All investigations \& procedures were done free of cost from the hospital with the help of Biochemistry \& Radiology department. Estimation of serum TSH, Prolactin, FBS, PPBS \& Husband Semen Analysis (after 3 days of abstinence) performed first. Serum FSH, LH \& AMH values were measured on day 2 of menstruation. HSG was performed at radiology department in an aseptic way. A water-soluble dye (Urografin) was used. The procedure was performed between days 6 and 10 of the menstrual cycle $\&$ at least 48 hours after menses had ceased. The women were advised to avoid unprotected intercourse in this period. A diagnostic laparoscopy was performed in the operating theatre under general anaesthesia, during the follicular phase of the menstrual cycle before the ovulatory period. Methylene 
blue was injected through an HSG cannula for chromopertubation. All the women were followed till definitive treatment for infertility, based on HSG and Laparoscopic findings.

Categorical variables are expressed as number \& percentage of patients. Sensitivity, specificity, positive predictive value (PPV), negative predictive value (NPV) studied \& compared. Chi-square was used to determine the concordance between the outcome of hysterosalpingography \& laparoscopy. The continuous variables were presented by means \pm standard deviation \& compared by using the independent sample $t$ test. The nonparametric variables \& data without normal distribution were tested by use of the Mann Whitney U test \& correlation analysis was performed by using Spearman Correlation test. The statistical software SPSS version 25 and Graph Pad Prism 5 has been used for analysis. An Alpha level of $5 \%$ has been taken, that means, all $p$ values $<0.05$ were considered statistically significant.

\section{RESULTS}

\section{Right Proximal Tubal Blockage}

At HSG blockage was present in $28(56 \%)$ patients whereas in DL, blockage was present in 24 (48\%) patients, which is significant.

\section{Right Distal Tubal Blockage}

At HSG blockage was in $20(40 \%)$ patients whereas in DL blockage was present in $14(28 \%)$ patients, which is significant.

\section{Left Proximal Tubal Blockage}

At HSG blockage was present in $16(32 \%)$ patients whereas in DL blockage was present in $20(40 \%)$ patients, which is significant.

\section{Left Distal Tubal Blockage}

At HSG blockage was present in $28(56 \%)$ patients whereas in DL blockage was present in 14 (28\%) patients and is statistically significant.

\section{Right Hydrosalpinx}

At HSG and DL hydrosalpinx was present in 16 (32\%) and $20(40 \%)$ patients respectively, which is significant.

\section{Left Hydrosalpinx}

At HSG and DL hydrosalpinx present in $6(12 \%)$ and $8(16 \%)$ patients respectively, which is significant.

\section{Right Peritubal Adhesion}

At HSG and DL per tubal adhesions are present in 18 (36\%) and 26 (52\%) patients respectively, which is significant.

\section{Left Peritubal Adhesion}

At HSG and DL adhesions were present in 10 (20\%) and 14 (28\%) patients respectively, which is significant.

Table 1 shows incidence of primary \& secondary infertility in the study population. It was $32(64 \%)$ \& 18 (36\%) cases respectively. Table 2 shows the incidence of infertility in different age group. It shows maximum cases (32/64\%) were found in the 30-35 yrs., of age group. Table 3 shows comparison of different tubal parameters in HSG \& Laparoscopy in the study population.

\begin{tabular}{|cccc|}
\hline Sl. No. & Infertility & Frequency & Incidence \% \\
1 & Primary & 32 & 64 \\
2 & Secondary & 18 & 36 \\
3 & Total & $\mathbf{5 0}$ & $\mathbf{1 0 0}$ \\
\hline \multicolumn{4}{r|}{ Table 1. Incidence of Infertility in the Study Population } \\
\hline
\end{tabular}

\begin{tabular}{|cccc|}
\hline Sl. No. & Age (Years) & Frequency & Incidence \% \\
1 & $20-25$ & 4 & 8 \\
2 & $25-30$ & 14 & 28 \\
3 & $30-35$ & 32 & 64 \\
4 & Total & $\mathbf{5 0}$ & $\mathbf{1 0 0}$ \\
\hline \multicolumn{2}{|c|}{ Table 2. Frequency Distribution of Age in the Study Population } \\
\hline
\end{tabular}

\begin{tabular}{|c|c|c|c|c|c|c|c|c|c|c|c|}
\hline हं & 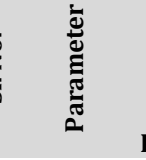 & HSG & LAP & HSG & 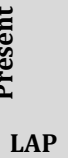 & 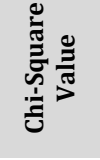 & 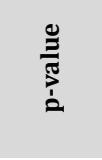 & 苞 & 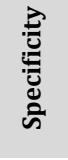 & $\vec{a}$ & 文 \\
\hline 1 & $\begin{array}{l}\text { Right prox. } \\
\text { tube }\end{array}$ & 22 & 26 & 28 & 24 & 36.2637 & $<0.0001$ & 84.6 & 100 & 100 & 85.7 \\
\hline 2 & $\begin{array}{c}\text { Right } \\
\text { dis tube }\end{array}$ & 30 & 36 & 20 & 14 & 29.1667 & 0.000001 & 84.6 & 100 & 100 & 70 \\
\hline 3 & $\begin{array}{l}\text { Left prox. } \\
\text { tube }\end{array}$ & 34 & 30 & 16 & 20 & 35.2941 & $<0.0001$ & 100 & 80 & 88.2 & 100 \\
\hline 4 & Left dis tube & 22 & 36 & 28 & 14 & 15.2778 & 0.00009 & 61.1 & 100 & 100 & 50 \\
\hline 5 & $\begin{array}{c}\text { Right } \\
\text { hydrosalpinx }\end{array}$ & 34 & 30 & 16 & 20 & 9632 & 0.0258 & 80 & 50 & 70.6 & 62.5 \\
\hline 6 & $\begin{array}{c}\text { Left } \\
\text { hydrosalpinx }\end{array}$ & 44 & 42 & 6 & 8 & 13.0231 & 0.0003 & 95.2 & 50 & 90.9 & 66.7 \\
\hline 7 & $\begin{array}{l}\text { Right } \\
\text { peritubal } \\
\text { adhesion }\end{array}$ & 32 & 24 & 18 & 26 & 4875 & .0062 & 83.3 & 53.8 & 62.5 & 77.8 \\
\hline 8 & $\begin{array}{c}\text { Left } \\
\text { peritubal } \\
\text { adhesion }\end{array}$ & 40 & 36 & 10 & 14 & 16.7659 & 0.00004 & 94,4 & 57.1 & 85 & 80 \\
\hline
\end{tabular}

\begin{tabular}{|ccccccc|}
\hline \multirow{2}{*}{ Sl. No. } & \multirow{2}{*}{ Parameter } & Absent & \multicolumn{2}{c|}{ Present } & Incidence \\
1 & Sub mucosal fibroid & 42 & 50 & 8 & 0 & 16 \\
2 & Intra mural fibroid & 50 & 44 & 0 & 6 & 12 \\
3 & Sub serous fibroid & 50 & 46 & 0 & 4 & 8 \\
4 & Endometrial polyp & 48 & 50 & 2 & 0 & 4 \\
5 & Intra uterine adhesion & 46 & 50 & 4 & 0 & 8 \\
\hline \multicolumn{6}{c}{ Table 4. Incidence of Uterine Pathology } \\
Detected by HSG \& Laparoscopy \\
\end{tabular}

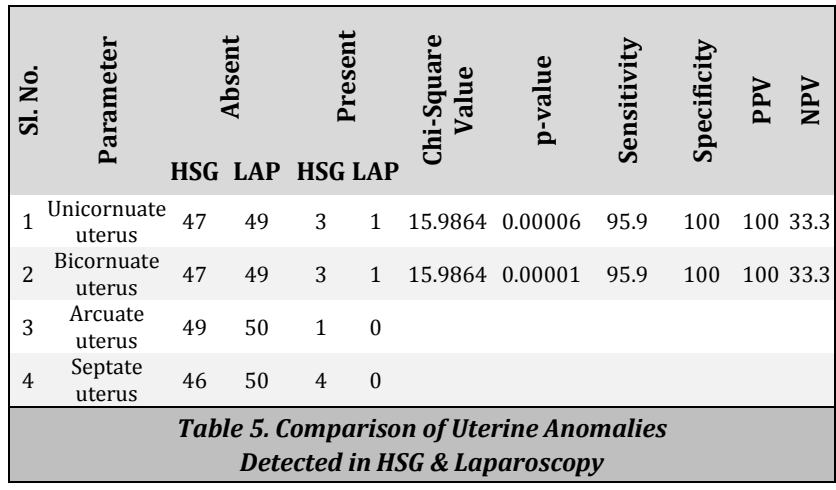

Table 4 is showing the incidence of uterine pathology detected by HSG \& Laparoscopy in the study population. Submucosal fibroids were detected by HSG in $16 \%$ cases and not detected by DL. Intramural fibroids were detected by Laparoscopy in $12 \%$ cases and not detected by HSG. Subserous fibroid was detected by laparoscopy in $8 \%$ cases and not detected by HSG. Incidence of Endometrial polyp and intrauterine adhesion in the study population were $4 \%$ and 
8\% respectively and detected only by HSG, not by Laparoscopy. Table 5 is showing the incidence and comparison of uterine anomalies detected in HSG \& Laparoscopy. Incidence of both unicornuate \& bicornuate uterus were same, which were $6 \%$ and $2 \%$ by HSG and DL respectively, and were statistically significant. Incidence of Arcuate uterus is $2 \%$ in both HSG and DL. Septate uterus is detected by HSG only in $8 \%$ cases.

\section{DISCUSSION}

Infertility is one of the most common disorder that a gynaecologist faces. The prevalence of infertility has increased in the last decade or so, because of an increase in stress, pelvic inflammatory diseases and increased tendency of delayed marriage \& childbearing. In the present study we compared HSG and laparoscopy in the diagnosis of tubal, peritubal \& uterine factors responsible for infertility. HSG is the initial investigation to assess the tubal patency as it is less invasive, cost effective and with less complication rate compared to laparoscopy. Laparoscopy has been suggested mandatory step for confirmation \& ruling out peritubal adhesion and endometriosis. ${ }^{9-12}$ Sensitivity \& specificity of an HSG are normally approximately $65 \%$ \& $85 \%$ respectively the reported sensitivity and specificity differed between studies concerning tubal occlusions.

\section{Types of Infertility}

In this study primary infertility was in $32(64 \%)$ patients and secondary infertility present in 18 patients (36\%).This finding is similar to the Iranian study by Seyedeh Zahra Masoumi ${ }^{12}$ et al during 2010 to 2011, where the incidence was $69.5 \%$ \& $30.5 \%$ respectively.

\section{Mean Age Group}

This study shows majority of participants (64\%) seeking medical help, due to infertility were between 30-35 years A study conducted in Cameroon by Jean Dupont Kemfens Ngowa ${ }^{13}$ showed that the mean age group seeking similar treatment was $31.4 \pm 6.4$ years. This is more or less similar \& signifies the treatment-seeking behavior of couples \& availability of health facilities.

\section{Tubal Blockage}

HSG \& DL identified blockage in Right proximal tube in 56\% \& $48 \%$ cases respectively). Sensitivity and specificity of HSG are 84.6 and 100.0; CI 95\% respectively and PPV and NPV are 100.0 and 85.7 respectively. Similarly, HSG estimation of Left proximal tubal blockage is $32 \%$ and $40 \%$ in laparoscopy. Sensitivity, specificity, PPV and NPV are 100.0, 80.0,88.2 and 100.0;CI 95\% respectively. Both false negative and positive results can be seen with HSG in the diagnosis of proximal tubal occlusion. ${ }^{14}$ The false positive result could be explained by the fact that spasm of uterine muscles during HSG following the injection of the contrast product may constrict or occlude one or both fallopian tubes. Small plugs of material, usually thought to be mucus or protein debris, can also occlude the proximal tube(s) where it is very narrow within the uterus. ${ }^{15}$
The false negative result can be explained by the fact that contrast intravasation into uterine and ovarian veins during HSG can sometimes be mistaken for tubal filling. ${ }^{16}$ The distal tubal occlusion is accessible to surgical therapeutic procedures and can lead to the practice of operative laparoscopy to improve fertility and prevent in vitro fertilization for some patients. ${ }^{17}$ In Right distal tube, HSG identified blockage in $40 \%$ and DL in $28 \%$ cases respectively. Sensitivity, specificity, PPV and NPV are 84.6, 100.0, 100.0, and 70.0 ; CI 95\% respectively. In left distal tube, HSG estimated $56 \%$ blockage whereas laparoscopy estimated a huge difference of 50 percent and detected blockage only in $28 \%$ cases. Sensitivity, specificity, PPV and NPV are 61.1, 100.0, 100.0 and 50.0;CI 95\% respectively.

There are also false negative and false positive distal tubal occlusions at HSG. The false positive results may be explained by the fact that in the presence of peritubal adhesions, even though the tubes may be patent, focal contrast deposits can lead to the misinterpretation as distal occlusions. ${ }^{11}$ Another explanation should be the faulty technique occurring while performing HSG. Insufficient pressure during uterine injection of contrast material due to vaginal reflux or the absence of the late radiographs for detection of pelvic diffusion of contrast material can lead to misdiagnosing as distal occlusion. On the contrary, the false negative tubal distal occlusion can be explained by the huge intravasation of contrast into pelvic veins which can be misinterpreted as tubal patency with peritoneal diffusion of contrast material. Another explanation is that, in case of one sided tubal distal occlusion, the pelvic diffusion of contrast materiel from one side can be misinterpreted as two sided tubal patency.

Another study showed HSG may be used as a screening test for tubal patency. Results demonstrates high specificity of HSG for diagnosis of tubal occlusion and low sensitivity in cases with peri-tubal adhesions ${ }^{9}$ In a research by Shah and et al 18 to evaluate and compare the diagnostic value of HSG and laparoscopy; HSG diagnosed bilateral proximal, bilateral distal and mixed tubal occlusion in $40.5 \%, 35.1 \%$ and $13.5 \%$ cases respectively. HSG demonstrated a reduced positive predictive value especially for bilateral proximal tubal occlusion. Results by Sakar ${ }^{8}$ et al. showed the sensitivity and specificity of HSG were $63 \%$ and $89.3 \%$ respectively, and the positive predictive value was $92 \%$, with a $55 \%$ negative predictive value, and the accuracy ratio was $72 \%$. In this study researchers concluded that these two methods are complementary.

\section{Hydrosalpinx}

By HSG Right sided hydrosalpinx were identified in $32 \%$ whereas in DL it was $40 \%$. Sensitivity, specificity, PPV and NPV are 80.0, 50.0, 70.6 and 62.5; CI 95\% respectively. HSG detected left sided hydrosalpinx in $12 \%$ and DL in $16 \%$ cases. Sensitivity, specificity, PPV and NPV are 95.2, 50.0, 90.9 and 66.7; CI 95\% respectively. The data shows that laparoscopy is a superior technique as far as detection of hydrosalpinx is concerned. The data is in agreement with a previous study that analysed the diagnostic value of laparoscopy in patients with tubal infertility. In that study tubal infertility diagnosed by laparoscopy accounted for 
$32.8 \%$ for infertile patients and the major causes of hydrosalpinx were pelvic tuberculosis and nonspecific inflammatory disease (NSID). The hydrosalpinx was particularly associated with the NSID group and in $70 \%$ of cases with NSID had complete tubular occlusion.

\section{Peritubal Adhesions}

Peri-tubal adhesions are a significant cause of infertility in women, altering the normal anatomic tubo-ovarian relationship and interfering the capture and transport of the ovum. ${ }^{19}$ In our study also showed that patients with secondary infections were more likely to have tubal adhesions. Though, HSG can detect peritubal adhesions, DL is superior for the detection of adhesions. Some of the factors associated with tubal adhesion include infection with Chlamydia and we therefore suspect that the role of PID in secondary infertility was under-reported in this study. This study therefore concluded that laparoscopy examination is a valuable procedure for the etiological diagnosis of tubal infertility. Another study suggests that the type of contrast media may affect the sensitivity of HSG in which case, watersoluble contrast media has better results and also has a therapeutic effect comparable to the oil-soluble contrast media. Right peritubal adhesion was $36 \%$ by HSG and by DL it was $52 \%$. Sensitivity, specificity, PPV and NPV are 83.3 , 53.8, 62.5 and 77.8; CI 95\% respectively. Similarly Left per tubal adhesion was $20 \%$ and $28 \%$ in HSG and DL respectively.

Sensitivity, specificity, PPV and NPV are 94.4, 57.1, 85.0 and 80.0; CI 95\% respectively. In a cohort study, eighty-two infertile cases were evaluated to compare tubo-peritoneal factors by HSG and laparoscopy. Results showed that pathological findings were observed in $45.1 \%$ by HSG and $65.85 \%$ by laparoscopy. The sensitivity and specificity of HSG were $63 \%$ and $89.3 \%$, and the positive predictive value was $92 \%$, with a $55 \%$ negative predictive value, and the accuracy ratio was $72 \% .{ }^{8}$ In above analysis we can observe that Right sided pathologies like blockage, hydrosalpinx and adhesions were detected more than left side both at HSG and DL. Increase incidence of such findings is due to presence of appendix, which has also been observed in other studies, while some studies did not detect the same finding. This study also observed that pathologies were more common in secondary infertility mainly in women with history of PID and dilatation and curettage history for incomplete abortion.

\section{Endometrial Polyp and Intrauterine Adhesions}

In present study detection by HSG are $4 \%$ and $8 \%$ respectively and only in women with secondary infertility. Intrauterine adhesion mainly presents in women who underwent intervention for abortion. Firoozeh Ahmadi et $\mathrm{al}^{20}$ noted the same association.

\section{Fibroids}

Many studies noted that in submucosal fibroid is mainly related to infertility and pregnancy loss. In present study, 16 $\%$ submucosal fibroids noted at HSG. In 2 patients of primary infertility and 6 patients of secondary infertility. Intramural and Subserous fibroids are noted at Laparoscopy were $12 \%$ and $8 \%$ respectively with more incidence in patients with secondary infertility. In present study unicornuate uterus detected at HSG and DL is $6 \%$ and 2\% respectively. Sensitivity, specificity, PPV and NPV are 95.9, 100.0, 100.0 and 33.3; CI 95\% respectively. Bicornuate uterus at HSG and DL was same $6 \%$ and $2 \%$ respectively. In the present study Arcuate uterus and Septate uterus only detected at HSG an are $\%$ and $8 \%$ respectively. In the present study the most common uterine anomaly detected was Septate uterus, while in another study by Bukar et al,21 bicornuate uterus was most common anomaly. Septate uterus was more common in women with secondary infertility with history of one or two pregnancy loss. Other studies corroborated the same.

\section{CONCLUSIONS}

HSG is a simple, easily available, and cost effective method for evaluation of tubal pathologies. It can also identify submucosal fibroids, endometrial polyp, intrauterine adhesions \& congenital uterine anomalies. It has a high specificity in diagnosing tubal blockage but low value in detecting tubal adhesion and hydrosalpinx. However, interpretation of HSG plates depends on the experience and ability of the radiologists involved. Laparoscopy is an appropriate method for examining the external part of fallopian tubes, fimbriae, tubo-ovarian relationship, adhesions and other pathologies such as endometriosis, tuberculosis \& other pelvic infections like chlamydial infection. The possible reasons for the difference in results between the two methods might be due to tubal spasm and endometrial polyp in the area of the uterine opening of the tubes \& anatomical variations in the tubes. Hysterosalpingography is useful in making decisions regarding further procedures like diagnostic laparoscopy for the diagnosis of infertility and should still serve as a useful primary investigation. These two methods are not alternatives, but are complementary in the diagnosis of tubal patency.

\section{ACKNOWLEDGEMENT}

Our sincerest thanks to Prof. Partha Mukhopadhay, HOD (G\&0), Eden Hospital, Medical College, Prof T. K. Lahiri, Principal, Medical College, Kolkata, Chairman, Institutional Ethics Committee, Medical College, \& all others who have made this possible.

\section{REFERENCES}

[1] Omoaregba J0, James BO, Lawani A0, et al. Psychosocial characteristics of female infertility in a tertiary health institution in Nigeria. Ann Afr Med 2011;10(1):19-24.

[2] Himmel W. Voluntary childlessness and being childfree. British Journal of General Practice 1997;47(1):11.

[3] Chowdhury SH, Cozma AI, Chowdhury JH. Infertility. Essentials for the Canadian medical licensing exam: review and prep for MCCQE Part I. $2^{\text {nd }}$ edn. Hong Kong: Wolters \& Kluwer 2017. 
[4] Bardawil T, Lucidi RS. "Fallopian Tube Disorders". Medscape Retrieved 2015.

[5] Adamson PC, Krupp K, Freeman AH, et al. Prevalence \& correlates of primary infertility among young women in Mysore, India. Indian J Med Res 2011;134(4):440-6.

[6] Sami N, Ali TS, Wasim S, et al. Risk factors for secondary infertility among women in Karachi, Pakistan. PLoS One 2012;7(4):e35828.

[7] Robabeh M, Roozbeh T. Comparison of hysterosalpingography and laparoscopy in infertile Iranian women with tubal factor. Ginekol Pol 2012;83(11):841-3.

[8] Sakar MN, Gul T, Atay AE, et al. Comparison of hysterosalpingography and laparoscopy in the evaluation of infertile women. Saudi Med J 2008;29(9):1315-8.

[9] Luttjeboer FY, Verhoeve HR, van Dessel HJ, et al. The value of medical history taking as risk indicator for tuboperitoneal pathology: a systematic review. BJOG 2009;116(5):612-25.

[10] Streda R, Mardesic T, Kult D, et al. The diagnostic value of hysterosalpingography in the diagnosis of tubal disease. Ceska Gynekol 2009;74(1):18-21.

[11] Tvarijonaviciene E, Nadisauskiene RJ. The value of hysterosalpingography in the diagnosis of tubal pathology among infertile patients. Medicina (Kaunas) 2008;44(6):439-48.

[12] Masoumi SZ, Parsa P, Darvish N, et al. An epidemiologic survey on the causes of infertility in patients referred to infertility center in Fatemieh Hospital in Hamadan. Iran J Reprod Med 2015;13(8):513-6.
[13] Ngowa JDK, Kasia JM, Georges NT, et al. Comparison of hysterosalpingograms with laparoscopy in the diagnostic of tubal factor of female infertility at the Yaoundé General Hospital, Cameroon. Pan Afr Med J 2015;22:264.

[14] Goynumer G, Yetim G, Gokcen 0 , et al. Hysterosalpingography, laparoscopy or both in the diagnosis of tubal disease in infertility. World $\mathrm{J}$ of Laparoscopic Surgery 2008;1(2):23-6.

[15] DeCherney AH. Anything you can do I can do better... or differently! Fertil Steril 1987;48(3):374-6.

[16] Siegler AM. Hysterosalpingography. Fertil Steril 1983;40(2):139-58.

[17] Taylor RC, Berkowitz J, McComb PF. Role of laparoscopic salpingostomy in the treatment of hydrosalpinx. Fertil Steril 2001;75(3):594-600.

[18] Shah SMH, Towobola OA, Masihleho M. Diagnosis of fallopian tube patency. East Afr Med J 2005;82(9):45762.

[19] Karasick S, Goldfarb AF. Peritubal adhesions in infertile women: diagnosis with hysterosalpingography. AJR Am J Roentgenol 1989;152(4):777-9.

[20] Ahmadi F, Rashidy Z, Haghighi H, et al. Uterine cavity assessment in infertile women: sensitivity and specificity of three-dimensional hysterosonography versus hysteroscopy. Iran J Reprod Med 2013;11(12):977-82.

[21] Bukar M, Mustapha Z, Takai UI, et al. Hysterosalpingographic findings in infertile women: a seven year review. Nigerian Journal of Clinical Practice 2011;14(2):168-70. 